\title{
UNCOUNTABILITY OF THE GROUP OF STRONG AUTOMORPHISMS OF WITT RING OF RATIONAL NUMBERS
}

\author{
Marcin Ryszard Stępień \\ Kielce University of Technology, Poland \\ mstepien@tu.kielce.pl
}

\begin{abstract}
We use the notion of rational self-equivalence which is a special case of Hilbertsymbol equivalence of fields, where both fields are considered to be the field $\mathbb{Q}$ of rational numbers. We define a small self-equivalence of the field $\mathbb{Q}$ as a special case of small equivalence of fields - a tool for constructing Hilbert-symbol equivalence of fields. We shall show, that one can choose initial sets of prime numbers and then control the processes of extending of small self-equivalence such that uncountable many rational self-equivalences can be constructed. The final conclusion is the corollary deciding that the group of strong automorphisms of Witt ring $W(\mathbb{Q})$ of rational numbers is uncountable.
\end{abstract}

\section{Introduction}

In this paper we shall show, how uncountalbly many strong automorphisms of Witt ring $W(\mathbb{Q})$ can be constructed.

We will use the notion of Hilbert-symbol equivalence of fields considered in [1], [2] and [3]. The ideas contained in these works are the base of our constructions. The restriction to the field $\mathbb{Q}$ of rational numbers allows us to simplify many details, therefore instead of frequent reference to literature we try to present the solution of the problem in the most complete way. In this section we shall outline the notions and facts which will be used in the paper. We use properties and facts from number theory, which can be found in [2], [4], [5] or [6].

Consider the field $\mathbb{Q}$ of rational numbers. Let IP denote the set of prime numbers together with the symbol $\infty$. In the follow we consider its two subsets:

$$
\mathbb{P}_{1}=\{p \in \mathbb{P} \backslash\{\infty\}: p \equiv 1(\bmod 4)\}, \quad \mathbb{P}_{\mathbf{3}}=\{p \in \mathbb{P} \backslash\{\infty\}: p \equiv 3(\bmod 4)\} .
$$

For each prime number $p$ there exists a completion $\mathbb{Q}_{p}$ of the field $\mathbb{Q}$ with respect to $p$-adic valuation $v_{p}$ called the field of $p$-adic numbers. Moreover we assume that $\mathbb{Q}_{\infty}=\mathbb{R}$ is a completion of $\mathbb{Q}$ with respect to usual absolute value (see [4]). Let $t$ be an automorphism of the group of square classes $t: \mathbb{Q} * / \mathbb{Q}^{*^{2}} \rightarrow \mathbb{Q} * \mathbb{Q}^{* 2}$ and let $T$ be an invertible map $T: \mathbb{P} \rightarrow \mathbb{P}$ preserving Hilbert symbols in the sense that $(a, b)_{p}=(t(a), t(b))_{T(p)}$ for all $a, b \in \mathbb{Q} * / \mathbb{Q}^{* 2}$ and for all $p \in \mathbb{P}$. The pair of maps 
$(T, t)$ we will call the Hilbert-symbol rational self-equivalence or shortly just rational self-equivalence. The notion of rational self-equivalence is a special case of a more general notion of Hilbert-symbol equivalence of fields, where the prime numbers are replaced by prime ideals of global fields (see [2,3]).

Two number fields have isomorphic Witt rings of quadratic forms if and only if there is a Hilbert-symbol equivalence between them (comp. [3]). Namely the bijection $t$ fulfilling the above conditions induces a strong isomorphism of Witt rings.

Constructing of Hilbert-symbol equivalence between Witt equivalent number fields is not an easy problem. The task of defining maps between infinite sets is difficult since there is no method of doing this in a finite number of steps. In [3] the authors reduced this problem to the problem of constructing so called small equivalence, which requires defining maps between finite sets of prime ideals. For more details the reader is referred to [3]. We use these ideas in order to construct the set of rational self-equivalences. There was shown in [7] that the set of rational self-equivalences is infinite and the effective construction of rational selfequivalences was presented. In this paper we shall prove that the set of rational self-equivalences is, in fact, uncountable.

Let $p$ and $q$ be two elements in the set IP. Every group isomorphism $t_{l o k}: \mathbb{Q}_{p}{ }^{*} / \mathbb{Q}_{p}{ }^{* 2} \rightarrow \mathbb{Q}_{q}{ }^{*} / \mathbb{Q}_{q}{ }^{2}$ preserving Hilbert symbols will be called local isomorphism. The local isomorphism $t_{l o k}$ is an isomorphism of quaternionic structures of local fields $\mathbb{Q}_{p}$ and $\mathbb{Q}_{q}$ (for more information about quaternionic structures and their isomorphisms see $[8,9])$. If $p$ and $q$ are prime numbers, then the local isomorphism $t_{l o k}: \mathbb{Q}_{p}{ }^{*} / \mathbb{Q}_{p}{ }^{* 2} \rightarrow \mathbb{Q}_{q}{ }^{*} / \mathbb{Q}_{q}{ }^{2}$ is called tame if $v_{q}\left(t_{l o k}(\mathrm{x})\right) \equiv v_{p}(x)(\bmod 2)$. It is well-known that if the prime numbers $p$ and $q$ are equivalent to 3 modulo 4, then -1 is not a square in fields $\mathbb{Q}_{p}$ and $\mathbb{Q}_{q}$ and every local isomorphism maps -1 to -1 . In this case every local isomorphism is tame. It is easy to notice that exactly two local isomorphisms exist for such a prime numbers $p$ and $q$. The first one fulfilling $t_{l o k}(p)=q$ we call simple local isomorphism and the other one $t_{l o k}(p)=-q$ we call skew local isomorphism.

By [3] p. 376 it follows that the rational self-equivalence $(T, t)$ determines the family of local isomorphisms $t_{p}: \mathbb{Q}_{p}{ }^{*} / \mathbb{Q}_{p}{ }^{2} \rightarrow \mathbb{Q}_{T(p)} * / \mathbb{Q}_{T(p)} *^{2}$ fulfilling the condition $t_{p}\left(a \mathbb{Q}_{p} *^{2}\right)=t(a) \mathbb{Q}_{T(p)} *^{2}$.

Let $S=\left\{p_{1}, \ldots, p_{k}\right\}$ be any finite subset of IP containing 2 and $\infty$. Let us fix that $p_{1}=\infty$ and $p_{2}=2$. We define the set of $S$-singular elements as follows:

$$
E_{S}=\left\{\mathrm{x} \in \mathbb{Q}^{*}: v_{p}(x) \equiv 0(\bmod 2) \text { for every } p \notin S\right\}
$$

Notice that $E_{S}$ is a subgroup of the multiplicative group of the field $\mathbb{Q}$ containing all squares of non-zero rational numbers. Therefore the quotient group $E_{S} / \mathbb{Q}^{* 2}$ is a subgroup of the group of square classes $\mathbb{Q} * / \mathbb{Q}^{* 2}$.

For any element $p \in \mathbb{P}$ the group $G_{p}=\mathbb{Q}_{p}{ }^{*} / \mathbb{Q}_{p}{ }^{2}$ (of exponent 2 ) can be viewed as a vector space over the two-element field $\mathrm{IF}_{2}$ and the Hilbert symbol determines 
non-degenerate bilinear form $\beta_{\mathrm{p}}: G_{p} \times G_{p} \rightarrow \mathbb{I}_{2}$ such that $(a, b)_{p}=(-1)^{\beta_{p}(a, b)}$. For given finite subset $S \subset \mathbb{P}$ we create a bilinear space $\left(G_{S}, \beta_{\mathrm{S}}\right)$, where

$$
G_{S}=\prod_{p \in S} G_{p}=\prod_{p \in S} \mathbb{Q}_{p}{ }^{*} / \mathbb{Q}_{p} *^{2} \quad \beta_{S}\left(\left[a_{p}\right]_{p \in S},\left[b_{p}\right]_{p \in S}\right)=\sum_{p \in S} \beta_{p}\left(a_{p}, b_{p}\right) .
$$

The space $\left(G_{S}, \beta_{S}\right)$ is an orthogonal sum of non-degenerate bilinear subspaces, hence it is also non-degenerate.

For every $p \in \mathbb{P}$ the natural imbedding of the field $\mathbb{Q}$ in $\mathbb{Q}_{p}$ induces the group homomorphism $i_{p}: \mathbb{Q} * / \mathbb{Q}^{* 2} \rightarrow \mathbb{Q}_{p} * / \mathbb{Q}_{p} *^{2}$ which is surjective. For given finite set $S=\left\{p_{1}, \ldots, p_{n}\right\} \subset \mathbb{P}$ we get the diagonal homomorphism $\operatorname{diag}_{S}: \mathbb{Q} * / \mathbb{Q}^{* 2} \rightarrow \mathrm{G}_{S}$ defined by $\operatorname{diag}_{S}\left(a \mathbb{Q}^{2}\right)=\left[i_{p_{l}}(a), \ldots, i_{p_{n}}(a)\right]=\left[a \mathbb{Q}_{p_{1}}^{* 2}, \ldots, a \mathbb{Q}_{p_{n}}^{*_{2}}\right]$ for all $\mathrm{a} \in \mathbb{Q}$. In order to simplify the notation the rational number $a$ will be often identified with its class of squares $a \mathbb{Q}^{*^{2}}$ and we will use the notation $\bar{a}=\operatorname{diag}_{S}\left(a \mathbb{Q}^{* 2}\right)$. The restriction of the homomorphism $\operatorname{diag}_{S}$ to the set of square classes of $S$-singular elements we denote by $i_{S}$.

Lemma 1.1. If $S=\left\{\infty, 2, p_{3}, \ldots, p_{n}\right\} \subset \mathbb{P}$, then

1. $\left\{-1 \mathbb{Q}^{* 2}, 2 \mathbb{Q}^{* 2}, p_{3} \mathbb{Q}^{* 2}, \ldots, p_{n} \mathbb{Q}^{* 2}\right\}$ is a basis of the space $E_{S} / \mathbb{Q}^{* 2}$.

2. $\operatorname{dim}\left(E_{S} / \mathbb{Q}^{*^{2}}\right)=|S|$.

3. $i_{S}$ is a group monomorphism.

4. $\operatorname{dim} G_{S}=2|S|$.

5. The subspace $i_{S}\left(E_{S} / \mathbb{Q}^{* 2}\right)$ is equal to its orthogonal completion in the linear space $\left(G_{S}, \beta_{S}\right)$.

Proof. By the definition of the set $E_{S}$ it follows that prime numbers which are not in $S$ can appear in decomposition of $x$ only with even exponents. Let $x=(-1)^{e_{1}} 2^{2 k_{2}+e_{2}} p_{3}^{2 k_{3}+e_{3}} \cdots p_{n}^{2 k_{n}+e_{n}} q_{1}^{2 l_{1}} \cdots q_{m}^{2 l_{m}}$ where $q_{1}, q_{2}, \ldots, q_{n} \notin S$ are prime numbers, $k_{i} l_{i} \in \mathbb{Z}$ and $e_{i} \in\{0,1\}$ be a canonical decomposition of any nonzero rational number $x$. Then $x \mathbb{Q}^{*^{2}}=(-1)^{e_{1}} 2^{e_{2}} p_{3}^{e_{3}} \cdots p_{n}^{e_{n}} \mathbb{Q}^{* 2}$. Hence the elements of the group $E_{S} / \mathbb{Q}^{*^{2}}$ are uniquely represented by integers of the form $(-1)^{e_{1}} 2^{e_{2}} p_{3}^{e_{3}} \cdots p_{n}^{e_{n}}$. In particular the elements $-1 \mathbb{Q} *^{2}, 2 \mathbb{Q} *^{2}, \ldots, \mathrm{p}_{3} \mathbb{Q}^{2}, \mathrm{p}_{n} \mathbb{Q}^{*^{2}}$ create a basis of linear space $E_{S} / \mathbb{Q}^{* 2}$ over $\mathbb{I}_{2}$ and $\operatorname{dim} E_{S} / \mathbb{Q}^{* 2}=|S|$. This finishes the proof of 1 and 2.

3. If $x \in \operatorname{ker} i_{S}$, then $x$ is a square in each $p$-adic field for $p \in \mathrm{S}$ and it follows that $x>0$ since the squares in the field $\mathbb{Q}_{\infty}=\mathbb{R}$ are positive numbers and $v_{p}(x) \equiv 0(\bmod 2)$ for prime numbers in $S$. It shows that $x$ is a product of prime 
numbers with even exponents, hence $x$ is a square of a rational number. Therefore ker $i_{S}=\left\{\mathbb{Q} *^{2}\right\}$.

4. It suffices to notice that $\operatorname{dim} G_{S}=\sum_{p \in S} \operatorname{dim} G_{p}=1+3+2(n-2)=2 n$ since $\operatorname{dim} G_{\infty}=\operatorname{dim} \mathbb{R} * / \mathbb{R}^{* 2}=1, \operatorname{dim} G_{2}=\operatorname{dim} \mathbb{Q}_{2} * / \mathbb{Q}_{2} *^{2}=3$ and $\operatorname{dim} G_{p}=\operatorname{dim} \mathbb{Q}_{p} * / \mathbb{Q}_{p} *^{2}=$ $=2$ for odd prime numbers $p$.

5. Let $x, y \in E_{S}$. By Hilbert reciprocity law $\prod_{p \in \mathbb{P}}(x, y)_{p}=1$ we get

$$
\prod_{p \in S}(x, y)_{p}=\prod_{p \in \mathbb{P} \backslash S}(x, y)_{p} .
$$

For every $p \in \mathbb{P} \backslash S$ the elements $x, y$ are $p$-adic units, hence $(x, y)_{p}=1$. Therefore

$$
(-1)^{\beta_{S}(x, y)}=\prod_{p \in S}(-1)^{\beta p(x, y)}=\prod_{p \in S}(x, y)_{p}=1
$$

Thus we get $\beta_{S}(x, y)=0$. It follows that for $F=i_{S}\left(E_{S} / \mathbb{Q}^{2}\right)$ we have $F \subseteq F^{\perp}$ and consequently $\operatorname{dim} F \leq \operatorname{dim} F^{\perp}$. Since $i_{S}$ is a monomorphism, hence $\operatorname{dim} F=$ $=\operatorname{dim} E_{S} / \mathbb{Q} *^{2}=|S|$. It is well-known that the bilinear space $\left(G_{S}, \beta_{S}\right)$ is nondegenerate, thus by the orthogonal complement theorem we get $\operatorname{dim} F^{\perp}=$ $=\operatorname{dim} G_{S}-\operatorname{dim} F=2|S|-|S|=n$. Since the subspaces $F$ and $F^{\perp}$ have the same dimensions and one of them is contained in the second one, thus $F=F^{\perp}$.

Definition 1.2. A small self-equivalence of the field $\mathbb{Q}$ defined on the set $S$ is a triplet $\mathfrak{R}=\left(S, T,\left\{t_{p}\right\}_{p \in S}\right)$ where

1) $S$ is a finite subset of $\mathrm{IP}$ and $\infty, 2 \in S$;

2) $T: S \rightarrow \mathbb{P}$ is an injection;

3) $\left\{t_{p}\right\}_{p \in S}$ is a family of local isomorphisms $t_{p}: \mathbb{Q}_{p} * / \mathbb{Q}_{p} *^{2} \rightarrow \mathbb{Q}_{T(p)} * / \mathbb{Q}_{T(p)} *^{2}$ preserving Hilbert symbols, i.e. $(a, b)_{p}=\left(t_{p}(a), t_{p}(b)\right)_{T(p)}$ for all $a, b \in \mathbb{Q}_{p} * / \mathbb{Q}_{p}{ }^{2}$.

The above definition of small self-equivalence imposes some restrictions on the map $T$. Namely $T(\infty)=\infty$, since $p=\infty$ is the only element of the set IP such that $\operatorname{dim} \mathbb{Q}_{p} * / \mathbb{Q}_{p}{ }^{2}=1$. Similarly $T(2)=2$, since $p=2$ is the only element of the set IP such that $\operatorname{dim} \mathbb{Q}_{p} * / \mathbb{Q}_{p}{ }^{2}=3$. Moreover by preserving the Hilbert symbol by the isomorphism $t_{p}: \mathbb{Q}_{p} * / \mathbb{Q}_{p} *^{2} \rightarrow \mathbb{Q}_{T(p)} * / \mathbb{Q}_{T(p)} *^{2}$ it follows that the quaternionic structures of the fields $\mathbb{Q}_{p}$ i $\mathbb{Q}_{T(p)}$ are isomorphic, what holds if and only if $T(p) \equiv p(\bmod$ 4). Conversely, if the injection $T: S \rightarrow \mathbb{P}$ fulfills these conditions, thus for any 
choice of local isomorphisms $t_{p}: \mathbb{Q}_{p} * / \mathbb{Q}_{p} *^{2} \rightarrow \mathbb{Q}_{T(p)} * / \mathbb{Q}_{T(p)} *^{2}$ for $p \in S$ the triplet $\left(S, T,\left\{t_{p}\right\}_{p \in S}\right)$ is a small self-equivalence.

Let us denote $S^{n}=T(S)$. By injectivity of the map $T$ it follows that the sets $S$ and $S$ have the same cardinality. A small self-equivalence defined on $S$ induces group isomorphism $\tau_{S}: G_{S} \rightarrow G_{S^{\prime}}$ which is a product of the family of local isomorphisms determined by the equivalence. If $\left[\alpha_{1}, \ldots, \alpha_{n}\right] \in G_{S}$, then $\tau_{S}\left(\left[\alpha_{1}, \ldots, \alpha_{n}\right]\right)=$ $=\left\lfloor t_{p_{1}}\left(\alpha_{1}\right), \ldots, t_{p_{n}}\left(\alpha_{n}\right)\right\rfloor$. Any small self-equivalence determines two sets

$$
\begin{aligned}
& H_{S}=\left\{\alpha \in E_{S} / \mathbb{Q}^{* 2}: \tau_{S}^{\circ} i_{S}(\alpha) \in i_{S}\left(E_{S}, / \mathbb{Q}^{* 2}\right)\right\} \subseteq E_{S} / \mathbb{Q}^{* 2}, \\
& H_{S^{\prime}}=\left\{\gamma \in E_{S^{\prime}} / \mathbb{Q}^{*^{2}}: \tau_{S}^{-1} \circ i_{S}(\gamma) \in i_{S}\left(E_{S} / \mathbb{Q}^{* 2}\right)\right\} \subseteq E_{S^{\prime}} / \mathbb{Q}^{* 2} .
\end{aligned}
$$

Since the maps $i_{S}, i_{S}, \tau_{S}$ are monomorphisms, then $t_{S}=\left.i_{S^{\prime}}^{-1} \circ \tau_{S} \circ i_{S}\right|_{H_{S}}$ maps $H_{S}$ isomorphically into $H_{S}$. The situation is presented on the following diagram

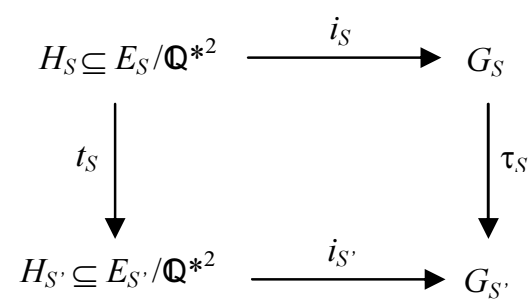

Lemma 1.3. For any small self-equivalence defined on the set $S$ the following conditions are equivalent:
1. $H_{S}=E_{S} / \mathbb{Q}^{2}$.
2. $H_{S^{\prime}}=E_{S^{\prime}} / \mathbb{Q}^{* 2}$
3. $\tau_{S} \circ i_{S}\left(E_{S} / \mathbb{Q} *^{2}\right)=i_{S}\left(E_{S}, / \mathbb{Q}^{* 2}\right)$.

Proof. It suffices to notice that by lemma $1.1 \operatorname{dim} i_{S}\left(E_{S} / \mathbb{Q} *{ }^{2}\right)=\operatorname{dim} i_{S}\left(E_{S^{\prime}} / \mathbb{Q} *^{2}\right)$, because the sets $S$ and $S^{\prime}$ are equinumerous.

If at least one of the conditions of the above lemma 1.3 is fulfilled, then the small self-equivalence $\mathfrak{R}$ will be called regular. In the other case we say that small self-equivalece is irregular and the number $\operatorname{def} \Re=\operatorname{dim} E_{S} / \mathbb{Q}^{* 2}-\operatorname{dim} H_{S}$ we call defect of small self-equivalence. If the small self-equivalence defined on the set $\mathrm{S}$ is regular, then $t_{S}$ is a group isomorphism between $E_{S} / \mathbb{Q}^{* 2}$ and $E_{S} / \mathbb{Q}^{* 2}$ and the equality $\tau_{S} \circ i_{S}=i_{S^{\prime}} \circ t_{S}$ holds.

Definition 1.4. We say that the small self-equivalence $\mathfrak{R}_{1}=\left(S_{1}, T_{1},\left\{t_{p}^{(1)}\right\}_{p \in S_{1}}\right)$ is an extension of the small self-equivalence $\mathfrak{R}=\left(S, T,\left\{t_{p}\right\}_{p \in S}\right)$ if 
1) $S \subseteq S_{1}$;

2) the map $T_{1}$ is an extension of the map $T$;

3) $t_{p}=t_{p}^{(1)}$ for all $p \in S$;

4) $H_{S} \subseteq H_{S_{1}}$ and the global isomorphism $t_{S_{1}}$ is an extension of the isomorphism $t_{S}$.

We say that the extension $\mathfrak{R}_{1}=\left(S_{1}, T_{1},\left\{t_{p}^{(1)}\right\}_{p \in S_{1}}\right)$ of the small self-equivalence $\mathfrak{R}=\left(S, T,\left\{t_{p}\right\}_{p \in S}\right)$ is determined by $q, q^{\prime} \in \mathbb{P}$ and the local isomorphism $t_{q}: \mathbb{Q}_{q} * / \mathbb{Q}_{q} *^{2} \rightarrow \mathbb{Q}_{q} * / \mathbb{Q}_{q} *^{2}$ where $S_{1}=S \cup\{q\}$ and $T_{1}$ is an extension of $T$ such that $T_{1}(q)=q^{\prime}$ and $\left\{t_{p}^{(1)}\right\}_{p \in S_{1}}=\left\{t_{p}\right\}_{p \in S} \cup\left\{t_{q}\right\}$.

We shall show that any regular small self-equivalence defined on arbitrary finite subset of IP containing 2 and $\infty$ can be extended to a rational self-equivalence. Next we will notice that the map $t: \mathbb{Q} * / \mathbb{Q} *^{2} \rightarrow \mathbb{Q} * / \mathbb{Q} *^{2}$ obtained in such a construction induces a strong automorphism of the Witt ring $W(\mathbb{Q})$ of rational numbers. We shall show how to control the construction in order to get uncountably many rational self-equivalences or uncountably many strong automorphisms of Witt ring $W(\mathbb{Q})$.

\section{The construction of rational self-equivalences}

Lemma 2.1 Assume that $S \subset \mathbb{P}$ is a finite set, $\infty, 2 \in S$ and $\alpha \in G_{S}$. Then there exists a prime number $q \notin S$ and an element $c \in E_{S}$ such that $\operatorname{diag}_{S}\left(c q \mathbb{Q} *^{2}\right)=\alpha$.

Proof. Let $a_{1}, \ldots, a_{n}$ be integers, which are not squares and let $\alpha=\left[a_{1} \mathbb{Q}_{p_{1}}^{*_{2}}\right.$, $\left.a_{2} \mathbb{Q}_{p_{2}}^{*_{2}}, \ldots, a_{n} \mathbb{Q}_{p_{n}}^{*_{2}}\right]$. We can assume that $a_{1} \in\{1,-1\}$. By the Chinese remainder theorem it follows that there exists a natural number $b$ such that $b \equiv a_{1} a_{2}(\bmod 16)$ and $b \equiv a_{1} a_{i}\left(\bmod p_{i}^{2}\right)$ for $i=3, \ldots, n$. Let us denote by $M=\left(4 p_{3} \cdots p_{n}\right)^{2}$ and $d=\operatorname{gcd}(b, M)$. Then the numbers $\frac{b}{d}$ and $\frac{M}{d}$ are coprime and by Dirichlet's theorem it follows that the arithmetic progression $\left(\frac{b}{d}+k \frac{M}{d}\right)_{\mathrm{k} \in \mathbb{N}}$ contains infinitely many prime numbers. Let $q$ be one such number coprime to $M$, thus $q \notin S$. Let us denote $a=a_{1} d q$. It is obvious that $a \equiv a_{1} b(\bmod M)$, hence $a \equiv a_{1} b\left(\bmod p_{i}^{2}\right)$ and $q d \equiv b(\bmod M)$ thus by transitivity of congruence relation we have $q d \equiv a_{1} a_{i}\left(\bmod p_{i}^{2}\right)$ and consequently $a_{1} d q \equiv a_{i}\left(\bmod p_{i}^{2}\right)$. Assume that $c=a_{1} d$. 
Therefore we have $c q \equiv a_{i}\left(\bmod p_{i}^{2}\right)$. Since $d \mid M$ hence $d \in E_{S}$ and it follows that the element $c=a_{1} d$ is in $E_{S}$.

Notice that the numbers $c q$ and $a_{l}$ are both positive or both negative. Moreover $v_{2}\left(a_{i}\right)=v_{2}(c q)<2,4 \leq v_{2}\left(c q-a_{2}\right)$ and $v_{p_{i}}\left(a_{i}\right)=v_{p_{i}}(c q)<2 \leq v_{p_{i}}\left(c q-a_{i}\right)$, then by Hensel's lemma it follows that $c q \mathbb{Q}_{p_{i}}^{*_{2}}=a_{i} \mathbb{Q}_{p_{i}}^{*_{2}}$ for $i=2, \ldots, n$. Therefore we get $i_{S}\left(c q \mathbb{Q}^{*}\right)=\alpha$.

Lemma 2.2. For any regular small self-equivalence $\mathfrak{R}=\left(S, T,\left\{t_{p}\right\}_{p \in S}\right)$ and for any prime number $q \in \mathbb{P} \backslash S$ there exists such a prime number $q^{\prime} \notin S^{\prime}$ that for any tame local isomorphism $t_{q}: G_{q} \rightarrow G_{q^{\prime}}$ the extension of the small self-equivalence $\Re$ determined by elements $q, q^{\prime}, t_{q}$ has a defect not bigger than 1. Moreover, there exists a tame local isomorphism $t_{q}^{\bullet}: G_{q} \rightarrow G_{q^{\prime}}$ such that the extension of selfequivalence $\mathfrak{R}$ determined by elements $q, q^{\prime}, t_{q}^{\bullet}$ is a regular small selfequivalence.

Proof. Let us fix $S^{\prime}=T(S)$ and $S_{1}=S \cup\{q\}$. If $\mathfrak{R}$ is a regular small selfequivalence, then $\tau_{S} \circ i_{S}=i_{S^{\prime}} \circ t_{S}$. Using lemma 2.1 there exists a prime number $q^{\prime} \notin S^{\prime}$ and an integer $a \in E_{S^{\prime}}$ such that $\overline{a q^{\prime}}=\tau_{S}(\bar{q})$. We define $S_{1}^{\prime}=S^{\prime} \cup\left\{q^{\prime}\right\}$ and $T_{1}(p)=T(p)$ for $p \in S$ and $T_{1}(q)=q^{\prime}$. Take any element $x \in E_{S}$. Since $\mathfrak{R}$ is regular, then there exists an element $x^{\prime} \in E_{S^{\prime}}$ such that $\tau_{S}(\bar{x})=\overline{x^{\prime}}$. Since $i_{S_{1}}(x)=\left[\bar{x}, i_{q}(x)\right] \in i_{S_{1}}\left(E_{S_{1}}\right)$, then according to lemma $1.1 \beta_{S_{1}}\left(\left[\bar{x}, i_{q}(x)\right],\left[\bar{q}, i_{q}(q)\right]\right)=$ $=\beta_{S}(\bar{x}, \bar{q})+\beta_{q}\left(i_{q}(x), i_{q}(q)\right)=0$. Similarly $i_{S_{1}^{\prime}}\left(x^{\prime}\right)=\left[\overline{x^{\prime}}, i_{q^{\prime}}\left(x^{\prime}\right)\right] \in i_{S_{1}^{\prime}}\left(E_{S_{1}^{\prime}}\right)$, hence according to lemma $1.1 \beta_{S_{1}^{\prime}}\left(\left[\overline{x^{\prime}}, i_{q^{\prime}}\left(x^{\prime}\right)\right],\left[\overline{q^{\prime}}, i_{q^{\prime}}\left(q^{\prime}\right)\right]\right)=\beta_{S^{\prime}}\left(\overline{x^{\prime}}, \overline{q^{\prime}}\right)+\beta_{q^{\prime}}\left(i_{q^{\prime}}\left(x^{\prime}\right), i_{q^{\prime}}\left(q^{\prime}\right)\right)=$ $=0$. Using the first formula and the fact that $\tau_{S}$ maps isometrically the space $\left(G_{S}, \beta_{S}\right)$ into $\left(G_{S^{\prime}}, \beta_{S^{\prime}}\right)$ we get $\beta_{q}(x, q)=\beta_{S}(\bar{x}, \bar{q})=\beta_{S^{\prime}}\left(\tau_{S}(\bar{x}), \tau_{S}(\bar{q})\right)=\beta_{S^{\prime}}\left(\overline{x^{\prime}}, \overline{a q^{\prime}}\right)$. Using again lemma 1.1 for elements $x^{\prime}, a \in E_{S^{\prime}}$ and the second formula above we get $\beta_{S^{\prime}}\left(\overline{x^{\prime}}, \overline{a q^{\prime}}\right)=\beta_{S^{\prime}}\left(\overline{x^{\prime}}, \bar{a}\right)+\beta_{S^{\prime}}\left(\overline{x^{\prime}}, \overline{q^{\prime}}\right)=\beta_{S^{\prime}}\left(\overline{x^{\prime}}, \overline{q^{\prime}}\right)=\beta_{q^{\prime}}\left(\overline{x^{\prime}}, \overline{q^{\prime}}\right)$. In this way we have shown that

$$
\beta_{q}(x, q)=\beta_{q^{\prime}}\left(x^{\prime}, q^{\prime}\right)
$$

In particular for $x=-1$ we have $\beta_{q}(-1, q)=\beta_{q^{\prime}}\left(-1, q^{\prime}\right)$, which means $\left(\frac{-1}{q}\right)=\left(\frac{-1}{q^{\prime}}\right)$, which is equivalent to $q \equiv q^{\prime}(\bmod 4)$. The last fact implies existing the local isomorphisms of groups of square classes of $q$-adic and $q$ '-adic fields $\mathbb{Q}_{q}{ }^{*} / \mathbb{Q}_{q}{ }^{2}$ and 
$\mathbb{Q}_{q} * / \mathbb{Q}_{q}, *^{2}$. Let $t_{q}: \mathbb{Q}_{q} * / \mathbb{Q}_{q} *^{2} \rightarrow \mathbb{Q}_{q} * / \mathbb{Q}_{q} *^{2}$ be any tame local isomorphism. By definition $t_{q}$ maps $q$-adic units to $q$ '-adic units. Moreover since $q$ is not a $q$-adic unit, then $t_{q}(q) \in\left\{q^{\prime}, u^{\prime} q^{\prime}\right\}$. We shall show that $q, q^{\prime}$ and $t_{q}$ determine the extension $\mathfrak{R}_{1}$ of the small self-equivalence $\mathfrak{R}$. We have to show, that $E_{S} / \mathbb{Q}^{2}=H_{S} \subseteq H_{S_{1}}$.

Since $\mathfrak{R}$ is regular, then the global isomorphism $t_{S}$ determined by $\mathfrak{R}$ is defined on whole group $E_{S} / \mathbb{Q}^{* 2}$. Take any element $x \in H_{S}=E_{S} / \mathbb{Q} *^{2}$. There are two cases possible: 1 . if $t_{q}(q)=q^{\prime}$, then $\beta_{q}(x, q)=\beta_{q^{\prime}}\left(t_{q}(x), t_{q}(q)\right)=\beta_{q^{\prime}}\left(t_{q}(x), q^{\prime}\right)$ and 2. if $t_{q}(q)=u^{\prime} q^{\prime}, \quad$ then $\quad \beta_{q}(x, q)=\beta_{q^{\prime}}\left(t_{q}(x), t_{q}(q)\right)=\beta_{q^{\prime}}\left(t_{q}(x), u^{\prime} q^{\prime}\right)=\beta_{q^{\prime}}\left(t_{q}(x), u^{\prime}\right)+$ $+\beta_{q^{\prime}}\left(t_{q}(x), q^{\prime}\right)=\beta_{q^{\prime}}\left(t_{q}(x), q^{\prime}\right)$, because $t_{q}(x)$ and $u^{\prime}$ are $q^{\prime}$-adic units. Therefore $\beta_{q^{\prime}}\left(t_{q}(x), u^{\prime}\right)=0$. Using the formula (1) for $x^{\prime}=t_{S}(x)$ in both cases we get $\beta_{q^{\prime}}\left(t_{S}(x), q^{\prime}\right)=\beta_{q^{\prime}}\left(t_{q}(x), q^{\prime}\right)$ and consequently $t_{S}(x)=t_{q}(x)$ in the group $\mathbb{Q}_{q^{\prime}} * / \mathbb{Q}_{q^{\prime}} *^{2}$. Therefore we have shown that $\tau_{S_{1}}\left(i_{S_{1}}(x)\right)=i_{S_{1}^{\prime}}\left(t_{S}(x)\right) \in i_{S^{\prime}}\left(E_{S^{\prime}} / \mathbb{Q}^{* 2}\right)$, hence $x \in H_{S_{1}}$. The defect of rational small self-equivalence $\mathfrak{R}_{1}$ is $\operatorname{def}\left(\Re_{1}\right)=\operatorname{dim} E_{S_{1}} / \mathbb{Q}^{2}-\operatorname{dim} H_{S_{1}} \leq \operatorname{dim} E_{S_{1}} / \mathbb{Q}^{* 2}-\operatorname{dim} H_{S}=\left|S_{1}\right|-|S|=1$.

Now we will show that for properly chosen local isomorphism we will get a regular extension of small self-equivalence $\mathfrak{R}$. Assume that $\mathbb{Q}_{q}{ }^{*} / \mathbb{Q}_{q} *^{2}=\{1, u, q, u q\}$ and $\mathbb{Q}_{q} * / \mathbb{Q}_{q} *^{2}=\left\{1, u^{\prime}, q^{\prime}, u^{\prime} q^{\prime}\right\}$. We define the local isomorphism $t_{q}^{\bullet}: \mathbb{Q}_{q} * / \mathbb{Q}_{q} *^{2} \rightarrow \mathbb{Q}_{q} * / \mathbb{Q}_{q}, *^{2}$ by $t_{q}^{\bullet}(u)=u^{\prime}$ and $t_{q}^{\bullet}(q)=a q^{\prime}$. The remaining values of $t_{q}^{\bullet}$ are uniquely determined: $t_{q}^{\bullet}(1)=1$ and $t_{q}^{\bullet}(u q)=u^{\prime} a q^{\prime}$. We can see that $t_{q}^{\bullet}$ is tame. If $a$ is a square in $\mathbb{Q}_{q}$, then $t_{q}^{\bullet}$ is a simple local isomorphism, in the other case $t_{q}^{\bullet}$ is a skew local isomorphism.

It is easy to notice that $E_{S_{1}} / \mathbb{Q}^{2}=E_{S} / \mathbb{Q}^{* 2} \cup q E_{S} / \mathbb{Q}^{* 2}$. In order to show that the small rational self-equivalence $\Re_{1}$ determined by $\Re$ and the elements $q, q^{\prime}$ and $t_{q}^{\bullet}$ is regular it suffices to check if $q \in H_{S 1}$, that means if the extension $t_{S_{1}}$ of global isomorphism $t_{S}$ can be defined for element $q \in E_{S 1} / \mathbb{Q} *^{2}$. Easy calculation $\tau_{S_{1}}\left(i_{S_{1}}(q)\right)=\tau_{S_{1}}([\bar{q}, q])=\left[\tau_{S}(\bar{q}), t_{q}^{\bullet}(q)\right]=\left[\overline{a q^{\prime}}, a q^{\prime}\right]=i_{S_{1}^{\prime}}\left(a q^{\prime}\right) \in i_{S_{1}^{\prime}}\left(E_{S_{1}^{\prime}}\right) \quad$ shows that $t_{S_{1}}(q)=a q^{\prime}$. In general $\tau_{S_{1}}\left(x q^{e}\right)=t_{S}(x)\left(a q^{\prime}\right)^{e}$ for all $x \in E_{S}$ and $e \in\{0,1\}$.

Lemma 2.3. For every rational small self-equivalence $\mathfrak{R}=\left(S, T,\left\{t_{p}\right\}_{p \in S}\right)$ with defect equal to 1 there exist prime numbers $q, q^{\prime} \in \mathbb{P}$ and a local isomorphism $t_{q}: G_{q} \rightarrow G_{q^{\prime}}$ such that the extension of $\mathfrak{R}$ determined by $q, q^{\prime}, t_{q}$ is a regular small self-equivalence. 
Proof. Let us denote by $S^{\prime}=T(S)$. Let $t_{S}$ be a fixed global monomorphism determined by $\mathfrak{R}$. According to hypothesis $\operatorname{dim} E_{S} / \mathbb{Q}^{* 2}-\operatorname{dim} H_{S}=1$, hence there exists $S$-singular element $x$ such that $\tau_{S}(\bar{x}) \notin i_{S^{\prime}}\left(E_{S} / \mathbb{Q}^{* 2}\right)$. By lemma 1.1 it follows that $i_{S},\left(E_{S}, / \mathbb{Q} *^{2}\right)$ equals to its orthogonal completion, hence every element, which does not belong to this set can not be orthogonal to all its elements. This means that there exists $S^{\prime}$-singular element $y \in \mathbb{Q}^{*}$ such that $\beta_{S^{\prime}}\left(\tau_{S}(\bar{x}), \bar{y}\right)=1$.

For any $z \in E_{S}$ we have $\beta_{S^{\prime}}\left(\tau_{S}(\bar{x}), \tau_{S}(\bar{z})\right)=\beta_{S}(\bar{x}, \bar{z})=0$, hence $y \notin H_{S^{\prime}}$. Next we have $\beta_{S}\left(\tau_{S}^{-1}(\bar{y}), \bar{x}\right)=\beta_{S^{\prime}}\left(\bar{y}, \tau_{S}(\bar{x})\right)=1$, thus $\tau_{S}^{-1}(\bar{y}) \notin i_{S}\left(E_{S} / \mathbb{Q} *^{2}\right)$. By lemma 2.1 there exists a prime number $q \notin S$ and the number $a \in E_{S}$, such that $\overline{a q}=\tau_{S}^{-1}(\bar{y})$. Similarly there exists a prime number $q^{\prime} \notin S^{\prime}$ and the number $a^{\prime} \in E_{S^{\prime}}$, such that $\overline{a^{\prime} q^{\prime}}=\tau_{S}(\bar{x})$. We define two sets $S_{1}=S \cup\{q\}$ and $S_{1}{ }^{\prime}=S^{\prime} \cup\left\{q^{\prime}\right\}$. First we notice that if $z \mathbb{Q}^{*^{2}} \in H_{S}$, then $z \in \mathbb{Q}_{q}{ }^{* 2}$. In fact, since $z$ and $a q$ are $S_{l}$-singular elements, therefore by lemma 1.1 it follows that $\beta_{S}(\bar{z}, \overline{a q})=0$ and consequently we get $\beta_{q}(z, a q)=\beta_{S}(\bar{z}, \overline{a q})=\beta_{S}\left(\bar{z}, \tau_{S}^{-1}(\bar{y})\right)=\beta_{S^{\prime}}\left(\tau_{S}(\bar{z}), \bar{y}\right)=0$. The last equality follows from $\tau_{S}(\bar{z}), \bar{y} \notin i_{S^{\prime}}\left(E_{S} / \mathbb{Q}^{* 2}\right)$. Since $z$ is $q$-adic unit modulo $\mathbb{Q}^{* 2}$, hence by equalities $v_{q}(a q)=1$ and $\beta_{q}(z, a q)=0$ it follows that $z \in \mathbb{Q}_{q}{ }^{2}$.

Analogously taking any $S^{\prime}$-singular element $z$ ' such that $z{ }^{\prime} \mathbb{Q}^{* 2} \in H_{S^{\prime}}$ and using equalities $\beta_{q^{\prime}}\left(z^{\prime}, a q^{\prime}\right)=\beta_{S^{\prime}}\left(\bar{z}, \tau_{S}(\bar{x})\right)=\beta_{S}\left(\tau_{S}^{-1}\left(z^{\prime}\right), \bar{x}\right)=0$ we find that $z^{\prime} \in \mathbb{Q}_{q} *^{2}$. Since $-1 \in H_{S}$ and $-1 \in H_{S^{\prime}}$, thus in particular it follows that $-1 \in \mathbb{Q}_{q}{ }^{2}$ and $-1 \in \mathbb{Q}_{q}, *^{2}$. This shows that $q, q^{\prime} \in \mathbb{P}_{1}$.

We extend the small self-equivalence $\mathfrak{R}$ to equivalence $\mathfrak{R}_{1}=\left(S_{1}, T_{1},\left\{t_{p}\right\}_{p \in S_{1}}\right)$ by extending bijection $T$ to the set $S_{l}$ and setting $T_{1}(q)=q^{\prime}$. It remains to define a local isomorphism $t_{q}: \mathbb{Q}_{q}{ }^{*} / \mathbb{Q}_{q}{ }^{2} \rightarrow \mathbb{Q}_{q} * / \mathbb{Q}_{q} *^{2}$. Let $u$ be a $q$-adic unit such that $\beta_{q}(u, a q)=1$ and let $u^{\prime}$ be a $q^{\prime}$-adic unit such that $\beta_{q^{\prime}}\left(u^{\prime}, a^{\prime} q^{\prime}\right)=1$. The classes of squares of elements $u$ and $a q$ make the basis of $\mathbb{Q}_{q}{ }^{*} / \mathbb{Q}_{q}{ }^{* 2}$ and the classes of squares of elements $u^{\prime}$ and $a^{\prime} q^{\prime}$ ' make the basis of $\mathbb{Q}_{q},{ }^{*} / \mathbb{Q}_{q},{ }^{2}$. We define the isomorphism $t_{q}$ by $t_{q}(u)=a^{\prime} q^{\prime}, t_{q}(a q)=u^{\prime}$.

Since -1 is a square in fields $\mathbb{Q}_{q}$ and $\mathbb{Q}_{q}$, it follows that every group isomorphism mapping the group $\mathbb{Q}_{q} * / \mathbb{Q}_{q}{ }^{* 2}$ into $\mathbb{Q}_{q} * / \mathbb{Q}_{q}, *^{2}$ preserves Hilbert symbols, thus in particular for $t_{q}$ defined above we have $(x, y)_{q}=\left(t_{q}(x), t_{q}(y)\right)_{q^{\prime}}$ for all $\mathrm{x}, \mathrm{y} \in \mathbb{Q}_{q}{ }^{*} / \mathbb{Q}_{q}{ }^{2}$. By adding $t_{q}$ to the family of local isomorphisms determined by the small self-equivalence $\mathfrak{R}$ we finish the construction of small self-equivalence $\mathfrak{R}_{1}$. 
It suffices to show that $\mathfrak{R}_{1}$ is an extension of $\mathfrak{R}$. Notice that $H_{S} \subset H_{S_{1}}$. In fact, let $z \mathbb{Q}^{* 2} \in H_{S}$ and let $t_{S}(z)=z^{\prime}$, that means $\tau_{S}(\bar{z})=\overline{z^{\prime}}$. Then $z^{\prime} \mathbb{Q}^{* 2} \in H_{S^{\prime}}$. Since $z=1$ in group $\mathbb{Q}_{q}{ }^{*} / \mathbb{Q}_{q}{ }^{* 2}$ and $z^{\prime}=1$ in $\mathbb{Q}_{q}{ }^{*} / \mathbb{Q}_{q}{ }^{* 2}$, then $t_{q}(z)=z^{\prime}$ and it follows $\tau_{S_{1}}(\bar{z})=\overline{z^{\prime}}$ in $G_{S_{1}}$ and consequently $z \mathbb{Q}^{* 2} \in H_{S_{1}}$.

It follows by above argumentation that if $z \mathbb{Q}^{* 2} \in H_{S}$, then $\tau_{S_{1}}(\bar{z})=\overline{\tau_{S}(z)}$. Therefore the global monomorphism $t_{S_{1}}$ for small self-equivalence $\mathfrak{R}_{1}$ is an extension of $t_{S}$. This finishes the proof that $\mathfrak{R}_{1}$ is an extension of $\mathfrak{R}$.

Now we shall show, that the square classes of elements $x$ and $a q$ are in $H_{S_{1}}$. Since $x$ and $a q$ are $S_{l^{-}}$-singular, then by lemma 1.1 we get equality $\beta_{S_{1}}(\bar{x}, \overline{a q})=0$. Thus we have $\beta_{q}(x, a q)=\beta_{S}(\bar{x}, \overline{a q})=\beta_{S}\left(\bar{x}, \tau_{S}^{-1}(\bar{y})\right)=\beta_{S^{\prime}}\left(\tau_{S}(\bar{x}), \bar{y}\right)=1$, hence $x=u$ in group $\mathbb{Q}_{q}{ }^{*} / \mathbb{Q}_{q}{ }^{2}$. By definition of $t_{q}$ we have $t_{q}(x)=a^{\prime} q^{\prime}$. With the fact $\tau_{S}(\bar{x})=\overline{a^{\prime} q^{\prime}}$ we get $\tau_{S_{1}}(\bar{x})=\overline{a^{\prime} q^{\prime}}$ in $G_{S_{1}^{\prime}}$. Now since $a^{\prime} q^{\prime} \in E_{S_{1}^{\prime}} / \mathbb{Q}_{q} *^{2}$, then $x \mathbb{Q}^{* 2} \in H_{S_{1}}$. Similarly $y$ and $a^{\prime} q^{\prime}$ are $S_{1}^{\prime}$-singular, then we have $\beta_{q^{\prime}}\left(y, a^{\prime} q^{\prime}\right)=\beta_{S^{\prime}}\left(\bar{y}, \overline{a^{\prime} q^{\prime}}\right)=\beta_{S^{\prime}}\left(\bar{y}, \tau_{S}(\bar{x})\right)=\beta_{S^{\prime}}\left(\tau_{S}(\bar{x}), \bar{y}\right)=1$. Therefore $y=u^{\prime}$ in the group $\mathbb{Q}_{q}, * / \mathbb{Q}_{q}, *^{2}$ and by the definition of isomorphism $t_{q}$ we have $a q=t_{q}^{-1}\left(u^{\prime}\right)=t_{q}^{-1}(y)$. On the other hand $\overline{a q}=\tau_{S}^{-1}(\bar{y})$, hence we get $\overline{a q}=\tau_{S_{1}}^{-1}(\bar{y})$ in $G_{S_{1}}$. The element $y$ is $S$-singular, thus it is $S_{l}$-singular too, hence $a q \mathbb{Q}^{* 2} \in H_{S_{1}}$. As a consequence we get the inclusion $H_{S} \cup\left\{x \mathbb{Q}^{* 2}, a q \mathbb{Q}^{* 2}\right\} \subset H_{S_{1}}$.

By definition of elements $x, a q$ it follows that $x \mathbb{Q}^{* 2} \in E_{S} / \mathbb{Q}^{*} \backslash H_{S}$ and $a q \mathbb{Q}^{* 2} \notin E_{S} / \mathbb{Q}^{* 2}$, hence $\operatorname{dim} H_{S_{1}} \geq \operatorname{dim} H_{S}+2$. The set $S_{l}$ was constructed by adding one prime number to the set $S$, thus $\operatorname{dim} E_{S_{1}} / \mathbb{Q}^{* 2}=\operatorname{dim} E_{S} / \mathbb{Q}^{* 2}+1$. Finally we get inequality $\operatorname{def} \mathfrak{R}_{S_{1}}=\operatorname{dim} E_{S_{1}} / \mathbb{Q}^{* 2}-\operatorname{dim} H_{S_{1}} \leq\left(\operatorname{dim} E_{S} / \mathbb{Q}^{* 2}+1\right)-\left(\operatorname{dim} H_{S}+2\right)=$ $=\operatorname{dim} E_{S} / \mathbb{Q}^{* 2}-\operatorname{dim} H_{S}-1=\operatorname{def} \Re_{S}-1=0$.

This finishes the proof of regularity of the rational small self-equivalence $\mathfrak{R}_{S_{1}}$.

Theorem 2.4. For every subset $A \subseteq \mathbb{P}_{3}$ there exists a rational self-equivalence $(T, t)$ such that induced local isomorphism $t_{q}: \mathbb{Q}_{q}{ }^{*} / \mathbb{Q}_{q}{ }^{2} \rightarrow \mathbb{Q}_{T(q)} * / \mathbb{Q}_{T(q)}{ }^{2}$ is simple (i. e. it fulfills condition $t_{q}\left(q \mathbb{Q}_{q}{ }^{2}\right)=T(q) \mathbb{Q}_{T(q)}{ }^{*^{2}}$ ) if and only if $q \in A$.

Proof. The rational self-equivalence will be constructed on some fixed small rational self-equivalence defined on the set $S=\{\infty, 2\}$ by adding suitable prime numbers. On each step of construction we have to control local isomorphisms as- 
sociated to prime numbers from $\mathbb{P}_{3}$. If starting small-equivalence is not regular, then by lemma 2.3 it can be extended to a regular small self-equivalence on the set $S$ enlarged with one prime number from the set $\mathbb{P}_{1}$. The small self-equivalence obtained in such a way we denote by $\mathfrak{R}_{0}=\left(S_{0}, T_{0},\left\{t_{p}\right\}_{p \in S_{0}}\right)$.

Assume that after $m$-th step we get a regular small self-equivalence $\mathfrak{R}_{m}=\left(S_{m}, T_{m},\left\{t_{p}\right\}_{p \in S_{m}}\right)$.

Step A. Let $q$ be the smallest prime number such that $q \notin S_{m}$. If $q \in \mathbb{P}_{1}$, then by lemma 2.2 there exists a prime number $q^{\prime} \in T_{m}\left(S_{m}\right)$ such that with the proper choice of local isomorphism $t_{q}$ the extension $\mathfrak{R}_{m+1}$ of the small self-equivalence $\mathfrak{R}_{m}$ determined by $q, q^{\prime}$ and $t_{q}$ will be a regular small self-equivalence.

If $q \in \mathbb{P}_{3}$, then by lemma $2.2 \mathfrak{R}_{m}$ can be extended to some small selfequivalence $\mathfrak{R}_{*}$ with any choice of local isomorphism $t_{q}$. Therefore if $q \in A$, then we assume that $t_{q}$ is a simple local isomorphism and in the other case we choose a skew local isomorphism for $t_{q}$. If the obtained extension is regular, then we take $\mathfrak{R}_{m+1}=\mathfrak{R}_{*}$ and we go to the next step. If the defect of small self-equivalence $\mathfrak{R}_{*}$ equals 1 , then by lemma 2.3 by adding suitable chosen prime numbers $q, q^{\prime} \in \mathbb{P}_{1}$ we get some regular small self-equivalence, which we denote by $\mathfrak{R}_{m+1}$.

Step B. We proceed as before with small self-equivalence inverse to $\mathfrak{R}_{m+1}$, which we denote $\mathfrak{R}^{\prime}=\left(S^{\prime}, T^{\prime},\left\{t_{p^{\prime}}^{\prime}\right\}_{p^{\prime} \in S^{\prime}}\right)$. We choose the smallest prime number $q^{\prime} \notin S^{\prime}$. As previously if $q^{\prime} \in \mathbb{P}_{1}$, then adding $q^{\prime}$ and properly chosen prime number $q \in \mathbb{P}_{1} \backslash T^{\prime}\left(S^{\prime}\right)$ to $\mathfrak{R}^{\prime}$ and suitable local isomorphism we get regular small selfequivalence $\mathfrak{R}^{\prime \prime}$.

If $q^{\prime} \in \mathbb{P}_{3} \backslash S^{\prime}$, then there exists a prime number $q \in \mathbb{P}_{3} \backslash T^{\prime}\left(S^{\prime}\right)$ which for any local isomorphism $t_{q^{\prime}}^{\prime}: G_{q^{\prime}} \rightarrow G_{q}$ gives the extension of small self-equivalence $\mathfrak{R}^{\prime}$ with a defect equal to 1 . Similarly as before we choose a simple local isomorphism if $q \in A$ and we choose a skew isomorphism in the other case. If the obtained extension is not regular, then by adding properly chosen prime numbers from $\mathbb{P}_{1}$ to the sets $S^{\prime}$ and $T^{\prime}\left(S^{\prime}\right)$ we get a regular small self-equivalence. Regular small selfequivalence $\mathfrak{R}^{\prime \prime}$ obtained in this step is an extension of small self-equivalence inverse to $\mathfrak{R}_{m+1}$. The small self-equivalence inverse to $\mathfrak{R}^{\prime \prime}$ we denote by $\mathfrak{R}_{m+2}$ and go to step A. Of course the small self-equivalence $\mathfrak{R}_{m+2}$ is an extension of $\mathfrak{R}_{m+1}$.

Continue this procedure we get one-to-one bijection of the set IP into itself. In order to define an automorphism $t$ of the group of square classes $\mathbb{Q}^{*} / \mathbb{Q}^{* 2}$ notice that every regular small self-equivalence $\left(S_{m}, T_{m},\left\{t_{p}\right\}_{p \in S_{m}}\right)$ defines isomorphism $t_{S_{m}}: E_{S_{m}} / \mathbb{Q}^{* 2} \rightarrow E_{S_{m}^{\prime}} / \mathbb{Q}^{* 2}$ preserving Hilbert symbols. Every nonzero rational 
number $a$ is a finite product of prime numbers with integer exponents, hence $a \in E_{S_{m}}$ for some $m \in \mathbb{N}$. It suffices to assume that $t\left(a \mathbb{Q}^{* 2}\right)=t_{S_{m}}\left(a \mathbb{Q}^{*^{2}}\right)$. It is obvious that the value of $t\left(a \mathbb{Q}^{*}\right)$ does not depend on the choice of $m$. In fact, assume that $a \in E_{S_{m_{1}}}$ and $a \in E_{S_{m_{2}}}$. We can assume that $m_{1} \leq m_{2}$. Then $E_{S_{m_{1}}} \subseteq E_{S_{m_{2}}}$ and by the fact that $t_{S_{m_{2}}}$ is an extension of $t_{S_{m_{1}}}$ we have $t_{S_{m_{2}}}\left(a \mathbb{Q} *{ }^{2}\right)=t_{S_{m_{1}}}\left(a \mathbb{Q} *^{2}\right)$.

We have shown that the pair $(T, t)$ is a rational self-equivalence.

Theorem 2.5. The group of strong automorphisms of Witt rings of rational numbers is uncountable.

Proof. It known (as follows from [1], [3] and [7]) that there exists one-to-one correspondence between rational self-equivalences and strong automorphisms of Witt rings (comp. [1],[3] and [7]). On the other hand every rational selfequivalence uniquely determines the set of this prime numbers with simple local isomorphisms. Since every subset (from the uncountably family of subsets) of $\mathrm{IP}_{3}$ is a set of prime numbers, which induces simple local isomorphisms, hence the group of strong automorphisms of Witt $\operatorname{ring} \mathrm{W}(\mathbb{Q})$ is uncountable.

\section{References}

[1] Czogała A., On reciprocity equivalence of quadratic number fields, Acta Arith. 1981, 58 (1), 27 46.

[2] Czogała A., Hilbert-symbol equivalence of global fields, Prace Naukowe Uniwersytetu Śląskiego w Katowicach vol. 1969, Wyd. Uniwersytetu Śląskiego, Katowice 2001 (in Polish).

[3] Czogała A., Hilbert-symbol equivalence of global function fields, Mathematica Slovaca 2001, 51, 4, 383-401.

[4] Browkin J., Field theory, Biblioteka Matematyczna 49, PWN, Warsaw 1978 (in Polish).

[5] Cassels J.W.S., Fröhlich A. (ed.), Algebraic Number Theory, Academic Press, London, New York 1967.

[6] Serre J.-P., A Course in Arithmetic, Springer-Verlag, New York, Heidelberg, Berlin 1973.

[7] Stępień M., A construction of infinite set of rational self-equivalences, Scientific Issues. Mathematics, XIV: 117-132, Jan Długosz University, Częstochowa 2009.

[8] Marshall M., Abstract Witt Rings, volume 57 of Queen's Papers in Pure and Applied Math. Queen's University, Ontario 1980

[9] Stępień M. R., Automorphisms of Witt rings and quaternionic structures, Scientific Research of the Institute of Mathematics and Computer Science Częstochowa University of Technology 2011, 1(10), 231-237. 